\title{
Análisis de la diversidad genética de 21 aislamientos del hongo Moniliophthora roreri basado en marcadores RAPD
}

\author{
Genetic diversity analysis of 21 Moniliophthora roreri fungus' \\ isolations based on RAPD markers
}

Boris A. Gutarra C. ${ }^{1}$, María J. Silva ${ }^{2}$, Kadir J. Márquez ${ }^{3}$, Betsabé León ${ }^{4}$ Instituto de Cultivos Tropicales, San Martín - Perú

\section{RESUMEN}

Objetivos: Estudiar la diversidad genética de 21 aislamientos del hongo que afecta al cultivo del cacao, Moniliophthora roreri, en tres zonas cacaoteras del Perú (Tocache, Mariscal Cáceres y Leoncio Prado). Métodos: Se utilizó 14 iniciadores RAPD (random amplified polymorphic DNA) polimórficos y una pareja de oligonucleótidos, los que fueron empleados bajo condiciones de amplificación estandarizadas. Con los datos obtenidos se construyó un dendograma utilizando el coeficiente de Jaccard y el algoritmo UPGMA (Unweighted Pair-Group Method using Arithmetic Average). La estructura genética fue estimada en función del análisis molecular de variancia (AMOVA) y la diversidad mediante los índices de Shannon y Nei. Resultados: Fueron conseguidas 59 bandas RAPD con un $73 \%$ de polimorfismo. El dendograma obtenido a un índice de similitud de 0,70 , claramente dividió los individuos en tres grupos. El análisis de la diversidad genética mostró altos valores en las zonas estudiadas de acuerdo con el índice de Shannon $(0,3936)$ y de Nei $(0,2622)$, con mayor riqueza en Leoncio Prado. Estas zonas presentan alta variabilidad, y según el AMOVA realizado: $88 \%$ entre accesiones por zona y solo $12 \%$ entre zonas. Conclusiones: Existe más de un grupo genético de Moniliophthora roreri en la Amazonía del Perú. Estos grupos, provenientes del Ecuador, pudieron haber ingresado por el intercambio de semillas $y / o$ de forma natural por medio de los ríos en común y estarían originando nuevos grupos genéticos locales.

1 Biólogo, M.Sc. en Biotecnología; responsable del laboratorio de Biología Molecular, Instituto de Cultivos Tropicales (San Martín, Perú). 2 Bachiller en Ciencias Biológicas. Universidad Particular Ricardo Palma.

3 Ingeniero agrónomo, M.Sc. en Fitopatología; responsable del laboratorio de Fitopatología, Instituto de Cultivos Tropicales (San Martín, Perú).

4 Ingeniero agrónomo; asistente de investigación del laboratorio de Fitopatología, Instituto de Cultivos Tropicales (San Martín, Perú). 


\section{ABSTRACT}

Objectives: To study the genetic diversity of 21 fungus' isolations that affect cocoa crops, Moniliophthora roreri in three cocoa areas (cacaoteras) from Peru (Tocache, Mariscal Cáceres and Leoncio Prado). Methods: 14 polymorphic RAPD indicators (random amplified polymorphic DNA) and an oligonucleotides pair were used, which were employed under standardized amplification terms. With the obtained data a dendrogram was constructed using the Jaccard coefficient and the UPGMA (Unweighted Pair-Group Method using Arithmetic Average) algorithm. The genetic structure was estimated based on the variance molecular analysis (AMOVA) and the diversity based on the Shannon and Nei indices. Results: We collected 59 RAPD bands with $73 \%$ of polymorphism. The obtained dendrogram at a similarity index of 0,70 , it clearly divided the individuals in three groups. The genetic diversity analysis showed high values in the three studied areas according to the Shannon index $(0,3936)$ and Nei $(0,2622)$, more richly in Leoncio Prado. The three areas have high variability within them, according to the performed AMOVA: $88 \%$ between accessions by area and only $12 \%$ between areas. Conclusions: The existence of more than one genetic group of Moniliophthora roreri in the Peru's Amazonia arises. These groups from Ecuador, may have entered by the exchange of seeds and/or in a natural way by rivers in common and they would be originating new local genetic groups.

Keywords: Moniliophthora roreri, Cacao, moniliasis, molecular markers.

\section{INTRODUCCIÓN}

Este estudio nace de la importancia de conocer cuál es la diversidad genética del fitopatógeno Moniliophthora roreri causante de la moniliasis, una de las enfermedades más destructivas del cacao, (Theobroma cacao) (figura $\mathrm{N}^{\circ}$ 1) (1). Como se sabe, este patógeno es endémico de la región amazónica y causa devastación al atacar los frutos del cacao. Algunos reportes científicos mencionan que ocasiona más del $30 \%$ de pérdidas en las cosechas, pero a veces la pérdida puede exceder el $90 \%$ bajo condiciones favorables de infestación (2). En el Perú más de 16500 hectáreas aproximadamente (más del $50 \%$ del área cultivada) ya fueron devastadas por este (3). Desde 1992, se ha detectado la presencia de moniliasis en el Alto Huallaga, en las provincias de Leoncio Prado (Huánuco) y Mariscal Cáceres (San Martin), así como en la región del Huallaga Central, caso que ocurre en la provincia de Tocache (San Martin) (4). Desde entonces la enfermedad ha sido continuamente observada por agricultores e investigadores.

El hongo Moniliophthora roreri es un basidiomiceto de la familia Marasmiaceae (1) que originalmente fue clasificado como un ascomiceto anamórfico (2) por su aparente ausencia del estado meiótico y ausencia de estructura sexual. Sin embargo, el advenimiento de nuevas tecnologías de observación de la ultra estructura citológica permitió concluir que el hongo debería ser reasignado como perteneciente a la división Basidiomycota (5). Más tarde, se reportó que podría existir un mecanismo citológico que permite que el hongo tenga reproducción sexual (6).

Algunos autores han advertido que a nivel de Latinoamérica Moniliophthora roreri estaría en una fase invasiva y se estaría dispersando de forma clonal (7). Según Phillips, 2003 (8), en Latinoamérica se ha determinado cinco grupos de Moniliophthora roreri, dos de ellos tienen amplia distribución, el grupo Bolívar (diseminado en el norte de Santander en Colombia, el oriente de Venezuela y los márgenes de Ecuador y Perú) y el grupo Co-occidental (en el lado occidental de Colombia, el centro de Ecuador y Centroamérica), y los otros tres son endémicos, el grupo Gileri (al noroeste de Ecuador) y los grupos Co-este y Cocentral (Colombia). 
En el Perú, Hernández et al (9) reportó por primera vez la presencia de Moniliophthora roreri en el departamento de Amazonas, caso que fue considerado como el primer reporte válido de la presencia del hongo en el Perú (10). En los años subsiguientes el patógeno fue reportado en el Alto Huallaga en el departamento de San Martin (11), en 1995 en el valle de Apurímac y en 1996 en el valle del Ene (12). En 1999 se detecta su presencia en el departamento del Cusco, así como en Jaén, Chachapoyas, San Martín, Huánuco y Ayacucho, de acuerdo con la información del Ministerio de Agricultura de Perú, aportada por Phillips (8). Este mismo año, a la vez, se señala que cerca del $99 \%$ de las plantaciones de cacao en el Perú diversidad genética de este en el Perú, que salvo el estudio de Phillips publicado en el 2003 (8) y el 2007 (2), no existen otros estudios sobre este aspecto plasmados con herramientas eficaces como los marcadores moleculares.

Esto no ha permitido tener certeza de cuántos grupos genéticos pudiesen existir y si es que el grupo denominado Bolívar es el único presente, hay que tener en cuenta que el estudio de Phillips et al 2003 (8) fue realizado con apenas cinco aislamientos de las localidades de Tingo María (Huánuco) y Tocache (San Martín), por lo que creemos que este es un aspecto no resuelto.

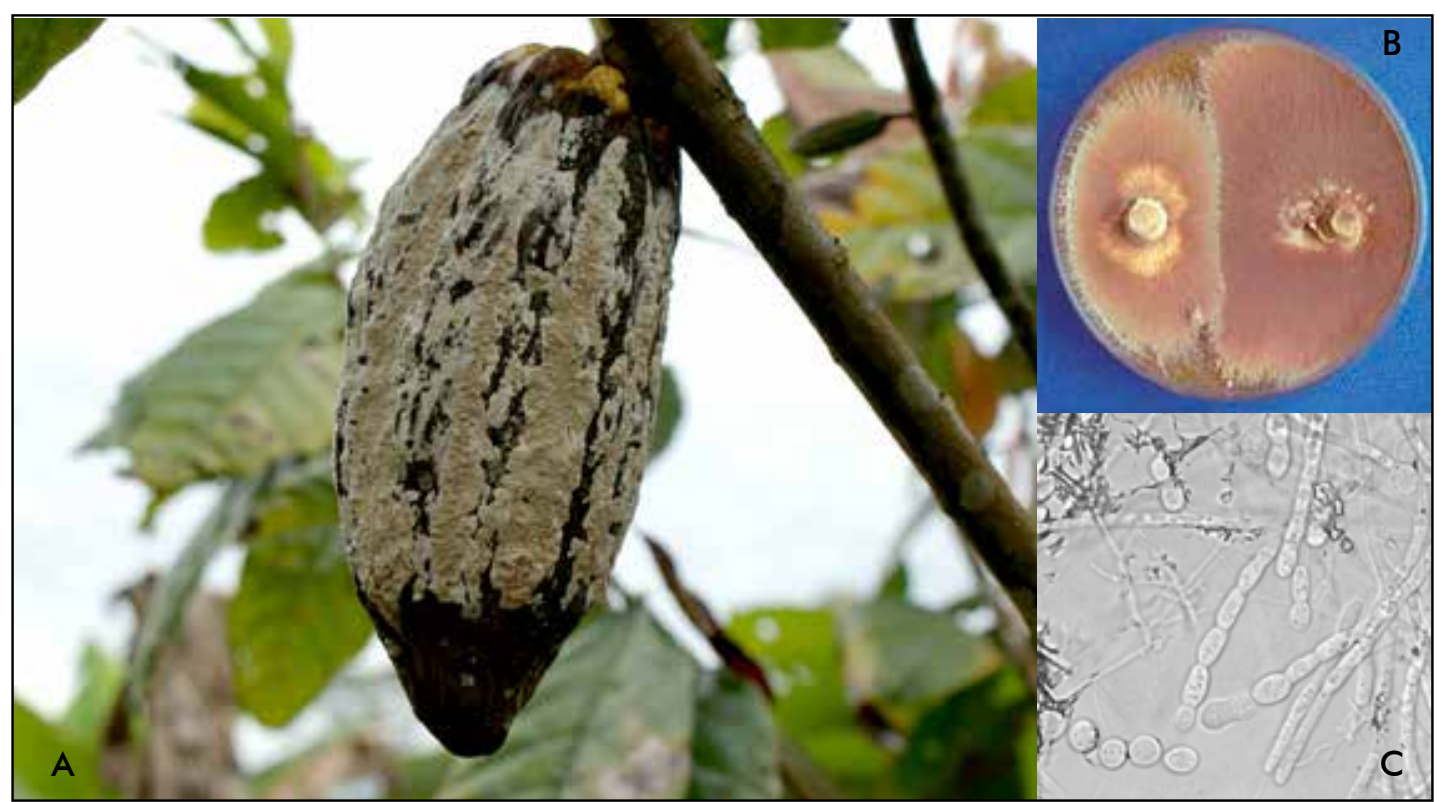

Figura № 1: A) Árbol de cacao (Theobroma cacao) infectado con M.roreri B) Aspecto macroscópico de colonias de M.roreri. C) Morfología microscópica de M.roreri.

están infectadas por Moniliophthora roreri y no existen barreras geográficas para su dispersión al departamento de Madre de Dios y de ahí al Brasil (13).

A pesar del impacto de Moniliophthora roreri en el Perú, hay muy pocos estudios realizados para conocer diferentes aspectos de la biología de este patógeno, limitándose a aspectos morfo fisiológicos y de control, entre ellos los estudios con biocontroladores como trichoderma endófitos (14) o el control mediante el uso de extractos vegetales (15). Muy poco se sabe, por ejemplo, sobre la
Un aspecto interesante en este hongo es la aparente facilidad de su genoma para adquirir mutaciones de forma rápida y responder a la presión de selección natural, asimismo su probable capacidad de reproducción sexual ha producido un desplazamiento rápido de Moniliophthora perniciosa, otro hongo patógeno de importancia para el cacao y estrechamente relacionado con el Moniliophthora roreri. (7)

El estudio de la diversidad genética es muy importante, ya que afecta diferentes 
aspectos de la biología del patógeno, incluyendo la relación patógeno-hospedero (2). La variación genética permite a los patógenos adaptarse rápidamente al aumento de la resistencia genética en los cultivos; de ahí que, este conocimiento sea crítico cuando se trata de desarrollar un programa de mejoramiento de cacao y búsqueda de clones resistentes.

Las provincias de Tocache, Mariscal Cáceres y Leoncio Prado contienen alta densidad de cultivos de cacao que muestran fuerte presencia de Moniliophthora roreri cuya diversidad genética ha sido muy poco estudiada. Por esta razón, el objetivo de la presente investigación es conocer la diversidad genética de este patógeno y determinar el número de grupos genéticos localizados en dichos lugares.

La metodología empleada tendrá como base los marcadores moleculares RAPD aplicados en 21 aislamientos colectados en estas provincias. La hipótesis es que existe alta diversidad genética y existirían más de un grupo genético de Moniliophthora roreri en las tres zonas de estudio. La investigación permitirá conocer la diversidad de este patógeno bajo condiciones nacionales y ayudará a trazar estrategias de control y selección de genotipos de cacao que muestren resistencia a grupos genéticos específicos de Moniliophthora roreri.

\section{MATERIAL Y MÉTODOS}

Se desarrolló una investigación con alcance explicativo que tuvo el siguiente procedimiento:

\section{Obtención de muestras de ADN}

Fueron colectadas muestras de frutos de cacao infectados con Moniliophthora roreri y se obtuvo el ADN de 21 aislamientos del referido hongo (tabla $N^{\circ} 1$ ), 6 de la provincia de Tocache (San Martín), 5 de la provincia de Mariscal Cáceres (San Martín) y 10 de la provincia de Leoncio Prado (Huánuco). Estas tres zonas con presencia de moniliasis (4). Para ello, se utilizó el ArchivePure DNA Cell/Tissue Kit de la compañía 5 PRIME, cuyo procedimiento aplicado fue en función de las instrucciones de los fabricantes.

\section{Iniciadores empleados}

Primero se ensayaron 19 iniciadores decaméricos (Operon Technologies Inc.), de los cuales solo 14 fueron seleccionados de acuerdo con su capacidad de generar bandas polimórficas y nítidas que se podían discriminar (tabla $\mathrm{N}^{\circ} 2$ ). Adicionalmente, se consideraron dos oligos (oligo 8 y oligo 10) empleados como iniciadores RAPD (7) en un estudio de variabilidad genética de Moniliophthora roreri. Es importante mencionar que no se conoce la procedencia de la secuencia de estos oligos.

\section{Análisis RAPD}

Se estandarizaron las condiciones térmicas de amplificación en el laboratorio eligiendo un programa RAPD para Moniliophthora roreri (7). Este consiste de un ciclo inicial de $95{ }^{\circ} \mathrm{C}$ x 5 minutos; luego 42 ciclos de 95 ${ }^{\circ} \mathrm{C}$ x 30 segundos, $36{ }^{\circ} \mathrm{C} \times 1$ minuto y 72 ${ }^{\circ} \mathrm{C} \times 2$ minutos; seguido de 1 ciclo de $72{ }^{\circ} \mathrm{C}$ x 5 minutos. En todos los casos la reacción de amplificación PCR consistió de: buffer de actividad PCR 1X, $2 \mathrm{mM}$ de $\mathrm{MgCl} 2,0,2 \mathrm{mM}$ de cada dNTPs, 0,2 mM de iniciador, 1,0 U de Taq polimerasa y $20 \mathrm{ng}$ de ADN en 15 $\mu \mathrm{L}$ de volumen final. Los productos de PCR fueron resueltos en geles de agarosa al 1,5 $\%$ y teñidos con bromuro de etidio al $1 \%$. La visualización se realizó en un transiluminador y la captura de imágenes con una cámara digital para su posterior análisis.

\section{Análisis de datos}

Para el análisis con marcadores RAPD, a los patrones de bandas obtenidos se le asignaron valores de 1 (presencia de banda) y 0 (ausencia de banda). En índice polimórfico (PIC), se empleó la fórmula $P I C=1-p 2-q 2$, donde $p$ es la frecuencia de banda y a la frecuencia de ausencia 
Tabla №1: Relación de aislamientos utilizados

\begin{tabular}{|c|c|c|c|c|c|}
\hline Departamento & Provincia (zona) & Código & Departamento & Provincia (zona) & Código \\
\hline San Martín & Tocache & Mrl & San Martín & Mariscal Cáceres & Mr11 \\
\hline San Martín & Tocache & Mr2 & Huánuco & Leoncio Prado & Mr12 \\
\hline San Martín & Tocache & Mr3 & Huánuco & Leoncio Prado & Mr13 \\
\hline San Martín & Tocache & Mr4 & Huánuco & Leoncio Prado & Mr14 \\
\hline San Martín & Tocache & Mr5 & Huánuco & Leoncio Prado & Mr15 \\
\hline San Martín & Tocache & Mr6 & Huánuco & Leoncio Prado & Mr16 \\
\hline San Martín & Mariscal Cáceres & Mr7 & Huánuco & Leoncio Prado & Mr17 \\
\hline San Martín & Mariscal Cáceres & Mr8 & Huánuco & Leoncio Prado & Mr18 \\
\hline San Martín & Mariscal Cáceres & Mr9 & Huánuco & Leoncio Prado & Mr19 \\
\hline \multirow[t]{2}{*}{ San Martín } & Mariscal Cáceres & Mr10 & Huánuco & Leoncio Prado & Mr20 \\
\hline & & & Huánuco & Leoncio Prado & Mr21 \\
\hline
\end{tabular}

*En el estudio las provincias son consideradas zonas porque el término "provincia" hace referencia a una división política.

Tabla № 2: Caracterización de los 14 iniciadores empleados en el análisis RAPD y el respectivo índice de marcador RAPD (IMR) alcanzado

\begin{tabular}{lccc}
\hline & & & \\
\hline Iniciador RAPD & BP/nTB & \% Poliformismo & IMR \\
\hline OLIGO8 & $1 / 4$ & 25 & 0,17 \\
OPA19 & $4 / 4$ & 100 & 0,96 \\
OPB5 & $3 / 4$ & 75 & 0,51 \\
OPC13 & $5 / 5$ & 100 & 1,67 \\
OLIGO10 & $2 / 4$ & 50 & 0,73 \\
OPA7 & $2 / 4$ & 50 & 0,72 \\
OPA17 & $4 / 5$ & 80 & 1,5 \\
OPB1 & $5 / 6$ & 83,3 & 1,9 \\
OPC1 & $4 / 5$ & 80 & 1,13 \\
OPA4 & $1 / 4$ & 25 & 0,44 \\
OPC2 & $2 / 3$ & 66 & 1,00 \\
OPE18 & $3 / 3$ & 100 & 0,98 \\
OPL5 & $4 / 4$ & 100 & 1,21 \\
OPE19 & 75 & 0,79 \\
Promedio total & $3 / 4$ & 72,1 & \\
\hline BP/nTB: Nún
\end{tabular}

BP/nTB: Número de bandas polimórficas entre el número total de bandas obtenidas por cada iniciador. IMR: Índice de marcador RAPD

de banda. Luego los valores PIC fueron usados para calcular el índice de marcador RAPD (IMR) (16). La distancia genética fue calculada con el coeficiente de Jaccard (17). Con las matrices generadas se realizó un análisis de agrupamiento mediante la técnica de ligamiento promedio UPGMA utilizando el programa NTSYS-pc programa versión 2.11. Con el fin de determinar la confiabilidad del dendrograma para representar la matriz de similitud original y poder visualizar las interrelaciones entre los aislamientos en tres dimensiones, se realizó un análisis de coordenadas principales
(PCoA)-3D empleando el programa FAMD (18). Ambos tipos de análisis se han utilizado para ordenar las unidades taxonómicas empleadas (OTUs) en diferentes trabajos de caracterización y diversidad molecular de diferentes especies $(2,19)$.

También se realizó el análisis cofénetico (Coph) para validar la confianza del análisis de agrupamiento empleando el módulo MXCOMP del programa NTSYS (20).

Para estimar la diversidad se calculó el índice de Shannon (I) y el índice de Nei (H) (21), 


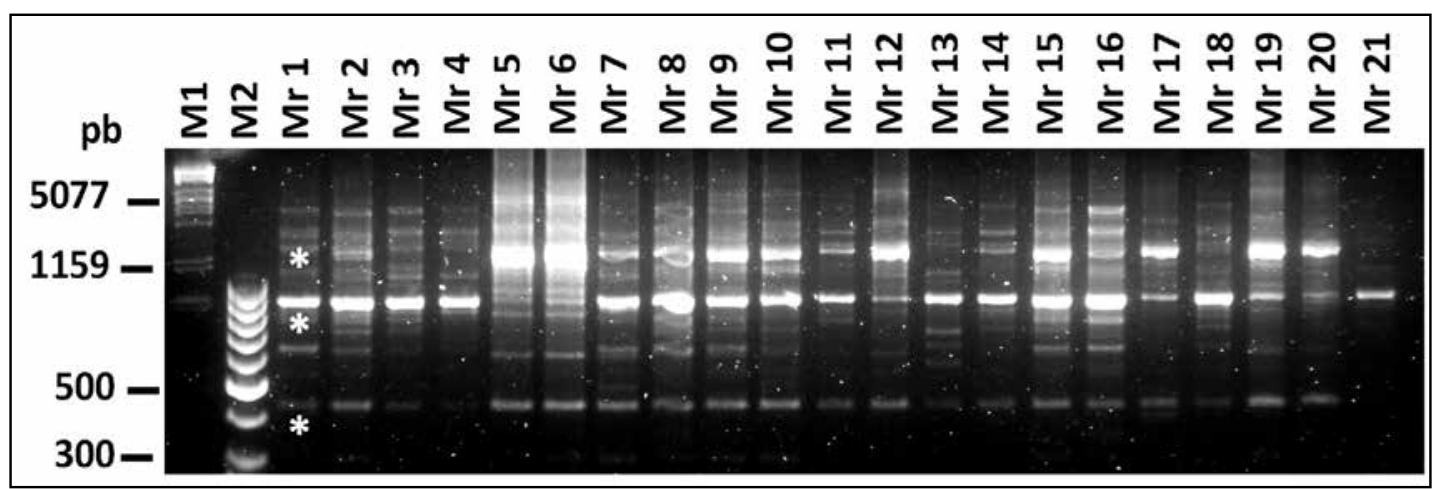

Figura № 2: Patrón de bandas RAPD con el iniciador OPC-13 en las muestras evaluadas. M1 representa el marcador $\lambda /$ Pstl y $M 2$ es el marcador $50 \mathrm{pb}$. Se puede distinguir el rango de tamaño para este iniciador (de 500 a 5077 pb aproximadamente) que muestran bandas claras y definidas. Los asteriscos muestran algunas bandas polimórficas.

el coeficiente de diferenciación genética (Gst) y el flujo genético $(\mathrm{Nm})$ utilizando el programa Popgene versión 1,31 (22).

Con estos mismos datos, se estimó la estructura genética en función del análisis molecular de variancia (AMOVA) a través de la diferenciación genética entre las zonas estudiadas. Este análisis fue con el programa GenAlEx 6.0 (23).

\section{RESULTADOS}

Los 14 iniciadores empleados generaron un total de 59 bandas claras y definidas con un promedio de 3,5 bandas por iniciador en un rango de 500 a $2000 \mathrm{pb}$, algunas de las cuales se aprecian en la figura $N^{\circ}$ 2. De estas bandas, $43(73 \%)$ fueron

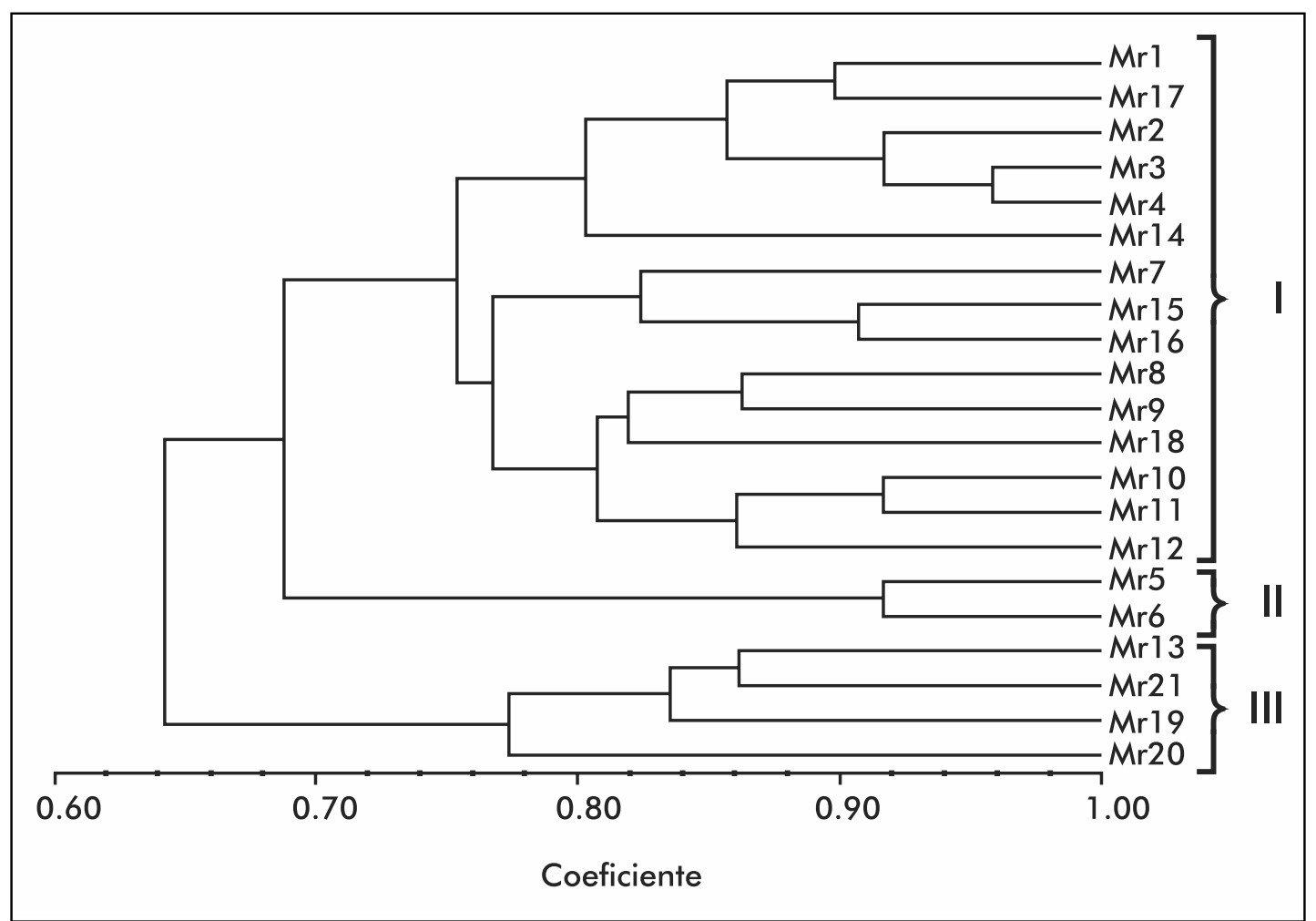

Figura № 3: Dendograma mostrando el parentesco entre los 21 aislamientos del hongo M. roreri con marcadores RAPD. A un índice de similitud de 0,7 se puede observar 3 grupos genéticos, indicados en números romanos (I- III) 


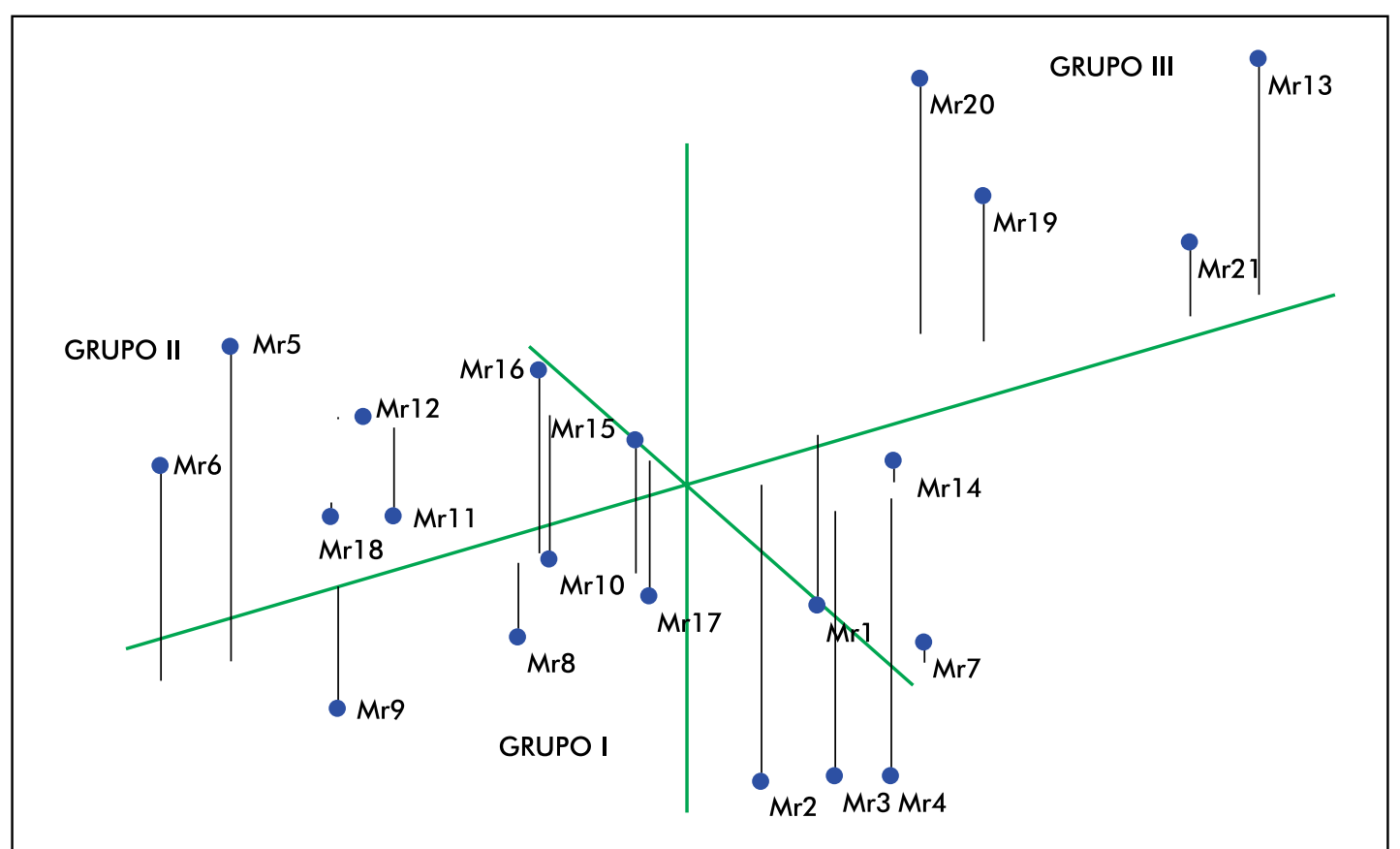

Figura № 4: Análisis de componentes principales (PCoA)-3D con ordenadas en "Y" basados en marcadores RAPD. Se puede notar el agrupamiento para los tres grupos genéticos (I, II y III)

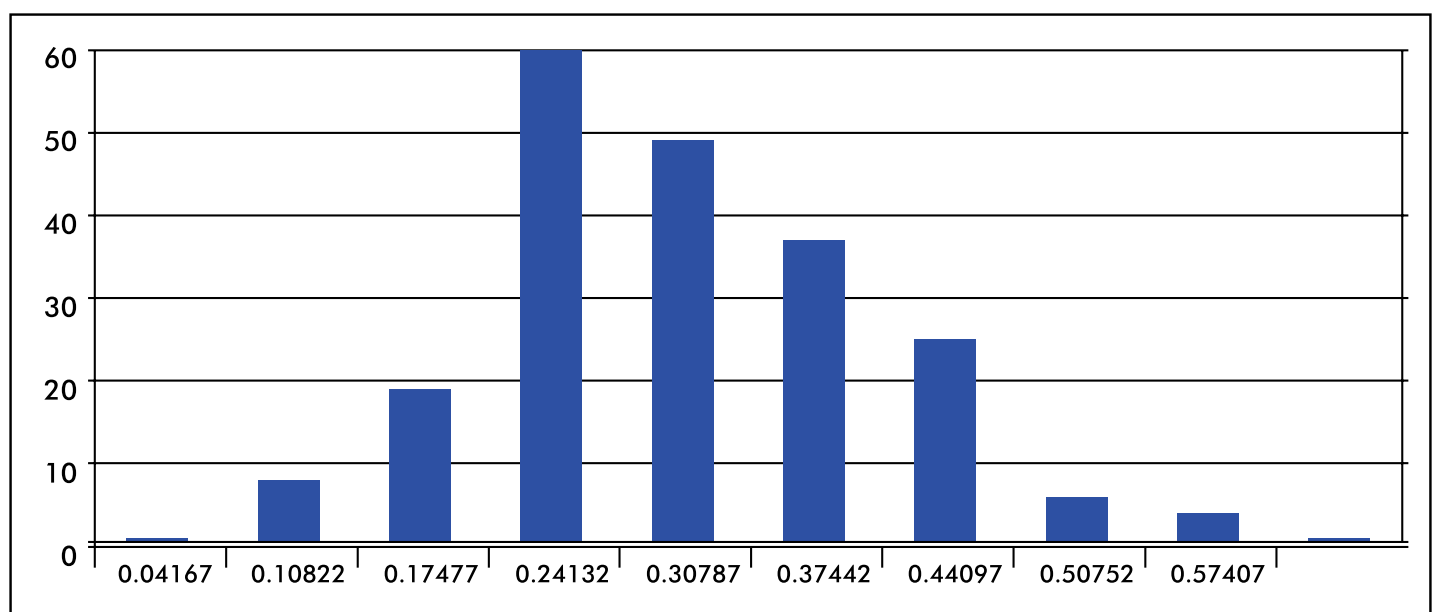

Figura № 5: Distribución de las distancias genéticas en función de marcadores RAPD entre los 21 aislamientos de Moniliophthora roreri. Se puede observar que la mayor distribución está entre $24,13 \%$ y $30,7 \%$

polimórficos y $16(27 \%)$ monomórficos. El índice de marcador RAPD (IMR) de cada uno de los marcadores empleados se puede observar en la tabla $N^{\circ} 2$. En ella se indica que los iniciadores OPB1, OPC13 y OPA 17 muestran los valores más altos $(1,9,1,67$ y 1,5 respectivamente). Estos iniciadores pueden ser utilizados en futuros estudios de diversidad e identificación genotípica del hongo Moniliophthora roreri debido a que en este tipo de estudios se privilegian los marcadores que otorgan mayor IMR (17).

Con un coeficiente de 0,70 el dendograma obtenido (figura $\mathrm{N}^{\circ} 3$ ) presenta tres grandes grupos. El primer grupo compuesto por los aislamientos Mr1, Mr17, Mr2, Mr3, Mr4, Mr14, Mr7, Mr15, Mr16, Mr8, Mr9, Mr18, Mr10, Mr11, Mr12; el segundo, por Mr5 y Mr6; y el tercero, por Mr20, Mr13, Mr21 y Mr19. El análisis de coordenadas principales (PCoA)-3D confirma este agrupamiento 
Tabla № 3: Comparación de la variación genética.

\begin{tabular}{lccccc}
\hline Zonas & & & & & \\
& PLP\% & Nei (h) & Shannon (I) & Gst & Nm \\
\hline Tocache & 45,76 & 0,1843 & 0,2695 & \\
Mariscal Cáceres & 37,29 & 0,1533 & 0,2218 & \\
Leoncio Prado & 57,63 & 0,2288 & 0,3352 & & \\
Entre poblaciones & & 0,2622 & 0,3963 & 0,2606 & 14,184 \\
\hline PLP: Porcentaje de loci polimórficos.
\end{tabular}

Tabla № 4: Porcentaje de variancia de los componentes del AMOVA.

\begin{tabular}{lccrrr}
\hline \multicolumn{7}{l}{ Fuente } & df & Sumas de cuadrados & MS & Est.variancia & \% de variancia \\
\hline Entre zonas & 2 & 24,710 & 12,35 & 0,861 & $12 \%$ \\
Accesiones/zonas & 18 & 119,1 & 6,61 & 6,61 & $88 \%$ \\
\hline Total & 20 & 143,810 & & 7,477 & $100 \%$ \\
\hline MS: Media de la suma de cuadrados & & & & \\
df: Grados de libertad & & & & \\
\hline
\end{tabular}

(figura $\mathrm{N}^{\circ} 4$ ). En promedio, las distancias genéticas fueron bajas $\left(27,4 \%\right.$ ) (figura $N^{\circ} 5$ ), la mayor distancia $(57,4 \%)$ ocurrió entre los aislamientos Mr13 y Mr6, y la menor (4,1\%), entre Mr4 y Mr3. El análisis cofenético presentó un $r=0,84$ que nos indica que hay una buena correlación entre el dendograma y la matriz de distancias.

El análisis de diversidad para los índices de Shannon (I) y Nei (H), así como el porcentaje de loci polimórficos, mostraron altos valores en las tres zonas estudiadas, pero los aislamientos de Mariscal Cáceres fueron los que mostraron mayor riqueza de diversidad genética de acuerdo con el índice de Shannon $(0,3)$ y el porcentaje de loci polimórficos (PLP) $\left(60,78 \%\right.$ ) (tabla $N^{\circ} 3$ ).

El análisis molecular de variancia (AMOVA) usando $p=0,01$, muestra que casi la totalidad de la variabilidad genética (88\%) es intrazonal; es decir, entre las accesiones por zona y no hay considerable diferencia entre las zonas evaluadas (tabla $\mathrm{N}^{\circ} 4$ ). El coeficiente de diferenciación genética fue significativo (Gst> 0,25) (24) presentando resultados similares al AMOVA ya que un Gst de 0,2606 indica que el 73,94\% de la diversidad radica dentro de la zonas (tabla $\mathrm{N}^{\circ}$ 3). El flujo genético (Nm) estimado obtenido fue de 1,4184 individuos por generación indicando que no hay efecto importante de la deriva genética y existiría intercambio genético entre aislamientos de estas zonas

\section{DISCUSIÓN}

En el estudio se determinó la presencia de más de un grupo en las tres regiones evaluadas, cuyos grupos además presentan una alta diversidad intrazonal como muestran los índices de diversidad y el AMOVA realizados. Estos datos, contradicen lo señalado por Phillips, 2003 (8), quien señala para el Perú la existencia de apenas un grupo genético al que denominan "Bolívar" y que provendría de la provincia de Napo, Ecuador. Según nuestros resultados, se plantea la hipótesis de que en el Perú existen más grupos que el denominado "Bolívar". Estos otros grupos pudieron haber ingresado al Perú por otros lugares, o sea a través de la provincia de San Ignacio, en el departamento de Cajamarca, promovido por el intercambio que se realizaba entre agricultores peruanos y ecuatorianos a inicios de los años 80 y también de forma natural por los ríos situados entre ambos países (25). De estos lugares, el hongo 
Moniliophthora roreri se habría diseminado por otras regiones del Perú.

Sin embargo, no podemos descartar que durante el proceso de selección natural, nuevos ambientes proporcionan la presión necesaria para enriquecer a poblaciones introducidas con nuevas variantes originadas por transferencia horizontal, fenómeno que ya ha demostrado su ocurrencia en Moniliophthora roreri y Moniliophthora perniciosa para la adquisición de genes involucrados en la fitopatogenicidad que podría estar permitiendo una rápida evolución de este hongo como ocurre con otros fitopatógenos $(26,27)$. A consecuencia de estos hechos, en el Perú, el hongo Moniliophthora roreri habría superado ya la fase puramente invasiva, ubicando sitios de evolución y focos de dispersión de nuevas variantes del hongo al igual que sucede en los municipios de Apartado y Dabeiba en Colombia (7).

La estructura genética es afectada por diferentes factores como el modo de reproducción, flujo genético, formas de dispersión y la selección natural (28). En este estudio se ha mostrado que a pesar de existir distancias considerables entre los puntos de colecta de los aislamientos que varían de 134 a 176 km la diferenciación de $12 \%$ como indica el AMOVA realizado entre estas poblaciones no es muy considerable. Una explicación sería el constante intercambio de frutos de cacao que se realizan entre los agricultores que de forma involuntaria estarían dispersando esporas de Moniliophthora roreri. Un valor de Gst de 0,2606 confirma que hay diferenciación entre las poblaciones y a diferencia de lo obtenido por Grisales y Afanador (7) cuyos valores fueron menores (osciló entre 0,12 y 0,25) para aislamientos de Moniliophthora roreri de Antioquia en Colombia indicarían que en estas zonas de estudio estaría actuando una presión de selección considerable haciendo que estas poblaciones no sean muy homogéneas. Generalmente un $\mathrm{Nm}>1$ es suficiente para superar los efectos de la deriva genética (29) evitándose así la perdida de la diversidad de estas poblaciones y además un flujo de 1,4184 aun sin ser muy considerable podría significar que no hay un aislamiento reproductivo entre los individuos y es posible la existencia de procesos de recombinación ya sea por mecanismos sexuales o transferencia horizontal entre algunos individuos de estas zonas.

Sobre la base de los resultados obtenidos, podemos concluir que en nuestro país se plantea la existencia de más de un grupo genético de Moniliophthora roreri, y que estos grupos, provenientes del Ecuador, posiblemente hayan ingresado por el intercambio de semillas entre agricultores de ambos países y/o de forma natural por medio de los ríos en común y podrían estar originado nuevos grupos genéticos al adaptarse a las nuevas condiciones medioambientales de la Amazonía peruana.

\section{Agradecimientos}

Esta investigación ha sido financiada por el Consejo Nacional de Ciencia, Tecnología e Innovación Tecnológica (CONCYTEC) en colaboración con el departamento de Agricultura de los Estados Unidos (USDA). Nuestro agradecimiento a Enrique Arévalo y Lucinda Vela por el apoyo en la búsqueda del financiamiento.

\section{REFERENCIAS BIBLIOGRÁFICAS}

1. Aime MC \& Phillips-Mora W. The causal agents of witches'broom and frosty pod rot of cacao (chocolate, Theobroma cacao) form a new lineage of Marasmiaceae. Mycologia. 2005; 97(5):1012-22.

2. Phillips MW, Aime MC y Wilkinson MJ. Biodiversity and biography of the cacao (Theobroma cacao) pathogen Moniliophthora roreri in tropical America. Plant Pathology. 2007; 56: 911-922.

3. Krauss U, Soberanis W. Rehabilitation of diseased cacao fields in Peru 
through shade regulation and timing of biocontrol measures. Agroforestry Systems. 2001; 53:179-184.

4. Programa de las Naciones Unidas para la Fiscalización Internacional de Drogas. Monilia del cacao. Detección, distribución y control en el Perú. Proyecto AD/PER/93/759-UNDCP-OSP; 1996. Boletín técnico.

5. Evans HC, Stalpers JA, Samson RA, Benny GL. On the taxonomy of Moniliophthora roreri, an important pathogen of Theobroma cacao in South America. Canadian Journal of Botany. 1978; 56 (20): 2528-2532.

6. Evans HC, Holmes KA, Phillips W, Wilkinson MJ. What's in a name: Crinipellis, the final resting place for the frosty pod rot pathogen of cocoa? Mycologist. 2002; 16: 148-52.

7. Grisales SP, Afanador LA. Análisis de variabilidad genética en Moniliophthora roreri con AP-PCR y RAPD en Antioquia, Colombia. Rev. Colomb. Biotecnol. 2007; 11(2): 15-32.

8. Phillips MW. Origin, biogeography, genetic diversity and taxonomic affinities of the cacao (Theobroma cacao) fungus Moniliophthora roreri (Cif.) Evans etál, as determined using molecular, phytopathological and morphophysiological evidence.Thesis for the degree of Doctor of Philosophy. Department of Agricultural Botany, School of Plant Sciences.The University of Reading, UK; 2003.

9. Hernández TA, Aranzazu F, Arévalo E \& Ríos R, 1990. La moniliasis del cacao en el Perú. Agrotrópica. 1990; 2: 56-58.

10. Evans HC, Krauss U, Ríos-Rutz R, Zecevich-Acosta T \& Arevalo-Gardini E. Cocoa in Peru. Cocoa Growers' Bulletin. 1998; 51: 7-22

11. Ríos R, Hernández TA \& Sánchez D, 1993. La Moniliasis (Moniliophthora roreri [Cif. and Par.] Evans) del Cacao en Juanjui-Saposoa. Directivas de Erradicación. Tropicultura. 1993; 5: 17 23.

12. Arévalo GE \& Ram A. La moniliasis del cacao en el Peru. Pura Selva.1997; 156: 35-39.

13. Arévalo-Gardini E. La moniliasis del cacao en el Perú (Reseña Histórica). Boletín del Instituto de Cultivos Tropicales San Martín, Perú. 1999; 2 p.

14. Arévalo E, Canto M, Leon B, Meinhard T. Biocontrol potencial de Moniliophthora roreri y Moniliophthora perniciosa con aislamientos de trichoderma endófito de cacao in vitro. Revista del XXI Congreso Peruano de Fitopatología, TarapotoPerú. 2010.

15. Leon $B$, Arévalo $E$, Márquez-Dávila K, Cayotopa J, Olivera D. Extractos de tara (Caesalpiniaspinosa) y matico (Piperaduncum) para el control de Moniliophthora roreri in vitro. Revista del XXI Congreso Peruano de Fitopatología, Tarapoto-Perú. 2010.

16. Raina SN, Rani V, Kojima T, Ogihara $Y$, Singh KP, Devarumath RM. RAPD and ISSR fingerprints as useful genetic markers for analysis of genetic diversity, varietal identification, and phylogenetic relationships in peanut (Arachishypogaea) cultivars and wild species. Genome. 2001; 44: 763-772.

17. Bonin A, Ehrich D \& Manel S. Statistical analysis of amplified fragment length polymorphism data: a toolbox for molecular ecologists and evolutionists. Molecular Ecology.2007; 16: 37373758.

18. Schlüter PM, Harris SA. Analysis of multilocus fingerprinting data sets containing missing data. Molecular Ecology Notes. 2006; 6(2):569-572.

19. De Freitas LB, Jerusalinsky L, Bonatto SL, Salzano, FM. Extreme homogeneity among brazilian wheat genotypes determined by RAPD markers. Pesq. agropec. bras. 2000; 35 (11): 2255 2260.

20. Rohlf FJ. NTSYS-PC: Numerical Taxonomy and Multivariate Analysis System Version 2.0. User Guide. New York: Applied Biostatistics Inc; 1998.

21. Nei M. Analysis of diversity in subdivided populations. Proc. Natl. Acad. Sci, USA. 1973; 70 (12): 3321-3323.

22. Yeh FC, Yang RC, Boyle TB, Ye ZH, Mao JX. Popgene version 1.31 Microsoft Window-based Freeware for Population Genetic Analysis.Quick User Guide. Alberta: University of Alberta And Tim 
Boyle, Centre for International Forestry Research; 1999.

23. Peakall R, Smouse PE. Genalex 6.0: genetic analysis in Excel. Population genetic software for teaching and research. MolEcol Notes. 2006; 6(1): 288-295

24. Slatkin M. Gene flow and geographic structure of natural populations. Science. 1987; 236: 787-792.

25. Evans HC, Krauss U, Ríos R, Zecevich $T$ y Arévalo E. Cocoa in Peru. Cocoa Growers' Bulletin. 1998; 51: 7-22.

26. Tiburcio RA, Lacerda GG, Falsarella $M$, Costa JM, Schuster SC, Carlson JE, et al. Genes acquired by horizontal transfer are potentially involved in the evolution of phytopathogenicity in Moniliophthora perniciosa and Moniliophthora roreri, two of the major pathogens of Cacao. J Mol Evol. 2010; 70 (1):85-97.

27. Brasier CM. Rapid evolution of introduced plant pathogens via interspecific hybridization. BioScience. 2001; 51(2):123-133.

28. Liu $Y Q$, Qin $L$, li $Y P$, Wang $H$, Xia RX, $\mathrm{Gi} Y \mathrm{H}$, et al. Comparative Genetic Diversity and Genetic Structure of Three Chinese Silkworm Species Bombyxmori L. (Lepidoptera: Bombycidae), AntheraeapernyiGuérin-Meneville and Samiacynthiaricini Donovan (Lepidoptera:Saturniidae). Neotropical Entomology. 2010; 39 (6): 967-975.

29. Allendorf FW. Gene flow and genetic differentiation among populations. ConservGenet. 1983; 18: 51-65 